differentiation of Th17 cells. In the nephrotoxic nephritis (NTN) mouse model of glomerulonephritis (GN), RGC-32 promotes organ damage through proinflammatory and profibrotic effects. To define the mechanisms underlying the role of RGC-32 in immune complex GN, we induced NTN in WT and RGC-32-/- mice.

Methods Proteinuria, blood urea nitrogen and kidney histopathology were determined to assess kidney damage. Single cell suspension of infiltrating kidneys and spleens cells were analyzed by flow cytometry and PCR for expression of IL-17A, CCR6, CCL20, CXCL1, CXCL2 and CXCL5.

Results Induction of NTN in RGC-32-/- mice resulted in decreased expression of IL-17A and CCR6 mRNA and decreased proportion of renal IL-17A+ CD4 $\mathrm{T}$ cells. The decrease in IL-17A+ CD4 cells was paralleled by decreased mRNA expression of CCL20 in both the renal tissue and infiltrating kidney cells of RGC-32-/- mice. In vitro, CD4+ T cells from spleens of RGC-32-/- mice cultured under Th17 conditions displayed decreased expression of CCL20 mRNA. These data suggest a role for RGC-32 in the positive feedback loop that enhances Th17 cell recruitment. The decrease in renal IL-17A + cells was associated with decreased expression of IL-17 induced chemokines CXCL1, CXCL2 and CXCL5 and decreased local infiltration of neutrophils.

Conclusions These data suggest a role for RGC-32 in the positive feedback loop that enhances Th17 cell recruitment. The decrease in renal IL-17A+ cells was associated with decreased expression of IL-17 induced chemokines CXCL1, CXCL2 and CXCL5 and decreased local infiltration of neutrophils.

\section{VOCLOSPORIN FOR LUPUS NEPHRITIS: INTERIM ANALYSIS OF THE AURORA 2 EXTENSION STUDY}

${ }^{1}$ Amit Saxena, ${ }^{2}$ Paola Mina-Osorio, ${ }^{2}$ Christopher Mela, ${ }^{2}$ Vanessa Birardi, ${ }^{2}$ Simrat Randhawa*. 'NYU Langone Health, USA; ${ }^{2}$ Aurinia Pharmaceuticals Inc., Canada

10.1136/lupus-2021-lupus21 century.32

Background Voclosporin, a novel calcineurin inhibitor, has been tested successfully in two pivotal trials in adult patients with lupus nephritis. The Phase 3 AURORA 1 study and the Phase 2 AURA-LV study showed that compared with mycophenolate mofetil (MMF) and low-dose steroids alone, the addition of voclosporin significantly increased the complete renal response rate at approximately one year of treatment. Patients that completed the AURORA 1 study were eligible to enroll in the AURORA 2 extension study. Here we report the first interim analysis of AURORA 2.

Methods AURORA 2 is a two-year, blinded, controlled extension study. Patients completing AURORA 1 continued the same randomized treatment in AURORA 2 of voclosporin (23.7 mg twice daily) or placebo, in combination with MMF (1 g twice daily) and low-dose steroids. The interim analysis evaluated urine protein creatinine ratio (UPCR) and estimated glomerular filtration rate (eGFR) in patients with up to two years of total treatment. In total, 116 patients in the voclosporin arm and 100 patients in the control arm enrolled in the extension study, of which 73 patients in the voclosporin arm and 51 patients in the control arm received two years of treatment at the time of this interim analysis.

Results Mean UPCR at pre-treatment AURORA 1 baseline was $3.9 \mathrm{mg} / \mathrm{mg}$ in the voclosporin arm $(\mathrm{n}=116)$ and $3.9 \mathrm{mg} / \mathrm{mg}$ in the control arm $(n=100)$. The least square (LS) mean change in UPCR from pre-treatment AURORA 1 baseline to year two was $-3.1 \mathrm{mg} / \mathrm{mg}$ for the voclosporin arm $(n=73)$ and $-2.1 \mathrm{mg} /$ $\mathrm{mg}$ for control arm $(\mathrm{n}=51$; figure 1$)$. Mean corrected eGFR at pre-treatment AURORA 1 baseline was $79.6 \mathrm{~mL} / \mathrm{min} / 1.73$ $\mathrm{m}^{2}$ for the voclosporin arm $(\mathrm{n}=116)$ and $78.9 \mathrm{~mL} / \mathrm{min} / 1.73$ $\mathrm{m}^{2}$ for the control arm $(\mathrm{n}=100)$ and at year two, was 79.0 $\mathrm{mL} / \mathrm{min} / 1.73 \mathrm{~m}^{2}$ for the voclosporin arm $(\mathrm{n}=73)$ and 82.9 $\mathrm{mL} / \mathrm{min} / 1.73 \mathrm{~m}^{2}$ for the control arm $(\mathrm{n}=51)$. There was a small, expected, and early decrease in mean eGFR in the voclosporin arm in the first four weeks of treatment (in AURORA 1) after which eGFR remained stable throughout year one and year two. Additionally, there were no unexpected new adverse events observed in patients who continued with voclosporin treatment compared to control-treated patients.

Mixed effects model for repeated measures (MMRM) analysis of least squares mean change from pre-treatment

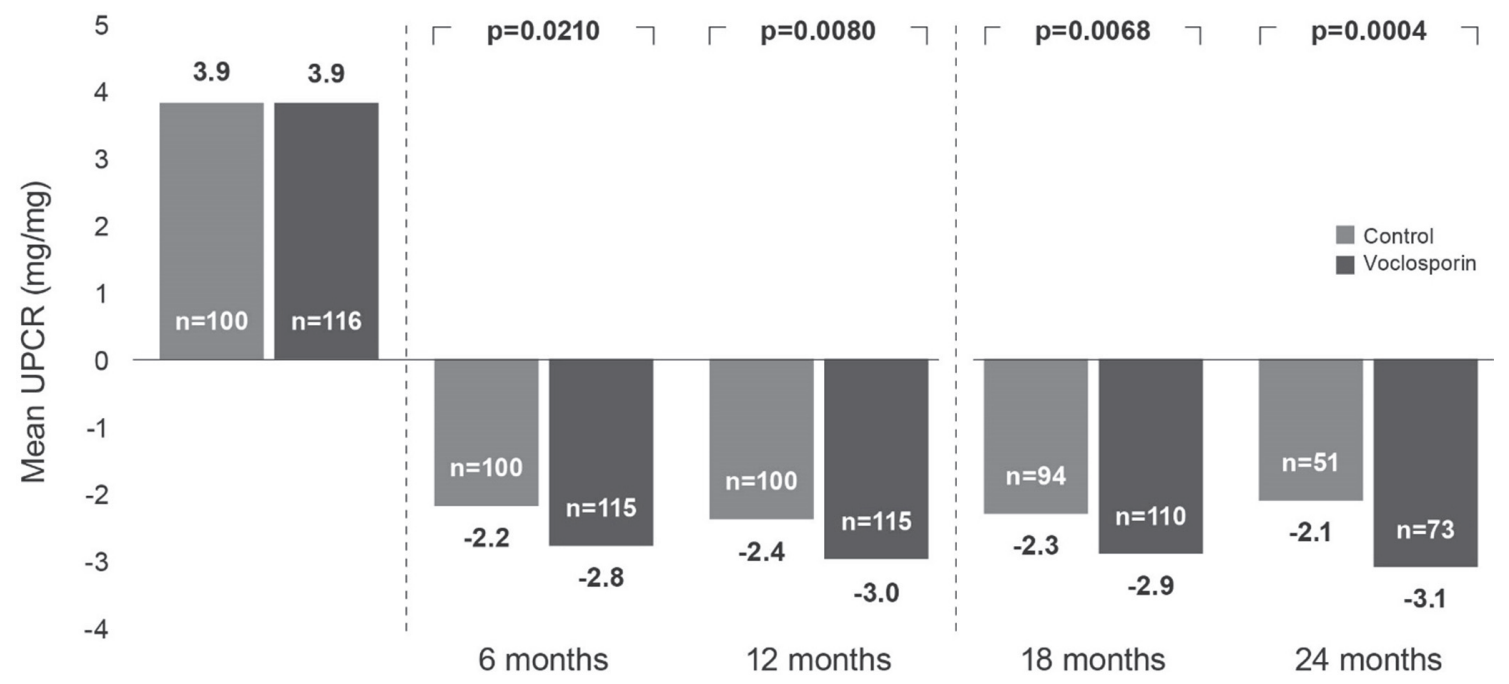
AURORA 1
AURORA 1
AURORA 2
Baseline

Abstract 514 Figure 1 UPCR change from baseline 
AURORA 1 baseline for UPCR included terms for baseline covariate, treatment, visit and treatment by visit interaction. Interim analysis of AURORA 2 patients includes data from pre-treatment baseline of AURORA 1, the one-year treatment period in AURORA 1 and up to one-year treatment period in AURORA 2.

Conclusions Patients in the voclosporin treatment arm maintained meaningful reductions in proteinuria with no change in mean eGFR at two years of treatment. Additional AURORA 2 efficacy and safety data will be provided at the conclusion of the study.

Acknowledgments Study funded by Aurinia Pharmaceuticals Inc.

Trial RegistrationClinicalTrials gov identifier: NCT03597464

\section{$600-T$ cells}

\section{AN IMBALANCE BETWEEN REGULATORY AND PRO- INFLAMMATORY T CELL SUBSETS DISTINGUISHES SYMPTOMATIC FROM ASYMPTOMATIC INDIVIDUALS WITH ANTI-NUCLEAR ANTIBODIES}

\begin{abstract}
${ }^{1}$ Emma Vanlieshout, ${ }^{1}$ Rashi Gupta, ${ }^{1}$ Kieran Manion, ${ }^{1}$ Dennisse Bonilla, ${ }^{1}$ Michael Kim, ${ }^{2}$ Sindhu R Johnson, ${ }^{3}$ Linda T Hiraki, ${ }^{2}$ Zareen Ahmad, ${ }^{1}, 4$ Zahi Touma, ${ }^{5}$ Arthur Bookman, 1,5Joan E Wither*. ${ }^{1}$ Schroeder Arthritis Institute, Krembil Research Institute, University Health Network, Toronto, Canada; ${ }^{2}$ Toronto Scleroderma Program, Division of Rheumatology, Toronto Western and Mount Sinai Hospitals, Toronto, Canada; ${ }^{3}$ Division of Rheumatology, The Hospital for Sick Children, Toronto, Canada; ${ }^{4}$ University of Toronto Lupus Clinic, Centre for Prognosis Studies in Rheumatic Diseases, Schroeder Arthritis Institute, University Health Network, Toronto, Canada; ${ }^{5}$ Division of Rheumatology, Schroeder Arthritis Institute, University Health Network, Toronto, Canada
\end{abstract}

10.1136/lupus-2021-lupus21century.33

Background $\mathrm{ANA}^{+}$systemic autoimmune rheumatic diseases (SARD), including SLE, have a prolonged pre-clinical phase during which ANAs can be detected in the absence of clinical symptoms. ANAs are also seen in healthy individuals, most of whom will not progress to SARD. The immunological changes that promote development of clinical symptoms in SARD and conversely maintain benign autoimmunity in asymptomatic $\mathrm{ANA}^{+}$individuals ( $\mathrm{ANA}^{+} \mathrm{NS}$ ) remain largely unexplored. To address this question, peripheral blood immune populations were examined in $\mathrm{ANA}^{+}$individuals, with and without SARD. Methods $\mathrm{ANA}^{+}$(IF $\left.\geq 1: 160\right)$ subjects were classified as $\mathrm{ANA}^{+} \mathrm{NS}(\mathrm{n}=61$, no SARD criteria), undifferentiated connective tissue disease (UCTD, $\mathrm{n}=54$, SARD criteria but lacking sufficient criteria for a diagnosis), or early SARD (SLE, $n=10$, $\mathrm{SjD}, \mathrm{n}=7, \mathrm{SSc}, \mathrm{n}=5$ ). All SARD patients were within 2 years of diagnosis and not taking Disease Modifying Anti-Rheumatic Drugs (hydroxychloroquine allowed) or prednisone. ANA ${ }^{-} \mathrm{HC}$ $(n=21)$ were also recruited. Peripheral blood mononuclear cells were isolated and stained with fluorochrome labeled antibodies to identify immune cell populations via flow cytometry. Plasma TGF-ß1 levels were measured by ELISA.

Results We previously showed that $\mathrm{ANA}^{+} \mathrm{NS}$ and UCTD patients have increased $\mathrm{B}$ cell activation and expansion of $\mathrm{T}$ follicular helper (Tfh) cells, similar to that seen in SARD. Here we show that $\mathrm{T}$ peripheral helper ( $\mathrm{Tph}$ ) cells are also increased in $\mathrm{ANA}^{+} \mathrm{NS}$ and demonstrate further progressive increases in UCTD and SARD, resulting in a significant increase in SARD relative to $\mathrm{ANA}^{+} \mathrm{NS}$. In $\mathrm{ANA}^{+} \mathrm{NS}$ and UCTD the majority of Tfh and Tph cells had a Th2 phenotype, leading to increased proportions of Tfh2 and Tph2 cells, as compared to $\mathrm{ANA}^{-} \mathrm{HC}$. In addition to these increases, SARD patients had significant elevations of Tph17 cells. Increases in Extrafollicular and Type 1 Regulatory $\mathrm{T}$ cells were also seen in $\mathrm{ANA}^{+} \mathrm{NS}$ and $\mathrm{UCTD}$, relative to $\mathrm{ANA}^{-} \mathrm{HC}$, with SARD patients demonstrating a trend to normalization of these populations. Similar changes were seen in the levels of TGF-ß1. ANA $^{+}$individuals who demonstrated symptomatic progression within the subsequent 2 years $(n=12)$ had significantly increased levels of activated class-switched and double negative memory $\mathrm{B}$ cells, plasmablasts, plasma cells, and $\mathrm{Tph}$ cells at baseline visit, as compared to those who remained stable over the same period of time.

Conclusion Collectively, our findings suggest active immunoregulation prevents clinical autoimmunity in $\mathrm{ANA}^{+} \mathrm{NS}$, and that this is impaired in patients with SARD, resulting in an imbalance between Tregs and pro-inflammatory $\mathrm{T}$ helper cell subsets.

\section{LONGITUDINAL CYTOF IMMUNOPHENOTYPING REVEALS DISTINCT PATTERNS OF T CELL-B CELL DYSREGULATION IN SLE}

${ }^{1}$ Takanori Sasaki, 'Sabrina Bracero, 'Lin Chen, ${ }^{1}$ Ye Cao, ${ }^{1}$ Emma Stevens, ${ }^{2} Y u j i e$ Qu, ${ }^{2}$ Guoxing Wang, ${ }^{1}$ Joshua Keegan, ${ }^{1}$ James A Lederer, ${ }^{2}$ Stephen E Alves, ${ }^{1}$ Karen H Costenbader, 'Deepak A Rao*. 'Brigham and Women's Hospital and Harvard Medical School, Boston, MA, USA; ${ }^{2}$ Merck and Co., Inc., Boston, MA, USA

\subsection{6/lupus-2021-lupus21century.34}

Background Mass cytometry (CyTOF) previously revealed that $\mathrm{T}$ follicular helper (Tfh) cells, $\mathrm{T}$ peripheral helper (Tph) cells, and age-associated B cells (ABCs) are robustly expanded in patients with newly diagnosed SLE. However, how these and other immune cell populations change over time in SLE remains unclear.

Methods We employed CyTOF with two 39 marker panels (T and B cell) in cryopreserved PBMCs from 9 newly diagnosed SLE, 15 established SLE, and 14 non-inflammatory controls. FlowSOM and marker analysis by tSNE used to identify and quantify clusters based on their 39-parameter characterization. For the newly diagnosed cohort, PBMCs were analyzed at 3 time points $(\mathrm{A}=$ at diagnosis, $\mathrm{B}=6$ months after the diagnosis, $\mathrm{C}=12$ months after the diagnosis). Serum samples were also analyzed to quantify 65 cytokines by Luminex multiplex assay, and associations between cell types and cytokines assessed by Spearman correlation.

Results We first confirmed that among CD4 T cells, Tfh cells (PD- $1^{\text {hi }}$ CXCR5 $^{+}$CD4 T cells) and Tph cells (PD-1 ${ }^{\text {hi }}$ CXCR5 CD4 $\mathrm{T}$ cells) were significantly increased in SLE patients. A broad analysis of $\mathrm{B}$ cells identified CD $11 \mathrm{c}^{+} \mathrm{CD} 21^{-} \mathrm{ABC}$ and CD19 ${ }^{\text {int }} \mathrm{Ki}^{\text {hi }} \mathrm{B}$ cell population significantly increased in the patients with SLE. This CD19 ${ }^{\text {int }} \mathrm{Ki} 67^{\text {hi }}$ cluster was also $\mathrm{CD} 21^{\text {lo }}, \mathrm{CD} 11 \mathrm{c}^{\text {low }}, \mathrm{CD} 27^{\text {hi }}$, and $\mathrm{CD} 38^{\text {hi }}$, consistent with a Ki67hi plasmablast population (figure 1A). Among CD8 T cells, we identified one highly expanded cluster in SLE patients compared to controls, which expressed $\mathrm{Ki}^{\mathrm{hi}}{ }^{\mathrm{hi}}$, ICOS ${ }^{\text {hi }}, \mathrm{PD}-1^{\text {int }}, \mathrm{CD} 57^{\text {low }}$, and granzyme $\mathrm{B}^{\text {int }}$ (figure $\left.1 \mathrm{~A}\right)$. In longitudinal analyses, the frequency of Tfh cells decreased over the first year of SLE, while Tph cells, ABCs, CD19 int Ki67 $7^{\text {hi }}$ plasmablasts, and $\mathrm{Ki}^{+} 7^{+} \mathrm{ICOS}^{+} \mathrm{CD} 8 \mathrm{~T}$ cells remained elevated at 12 months (figure 1B). Correlation analyses including both immune cell frequencies and cytokines revealed an association of Tph cells, Ki67 ${ }^{+} \mathrm{ICOS}^{+} \mathrm{CD} 8 \mathrm{~T}$ cells, ABCs, 